\title{
Análise do uso de antibióticos na profilaxia de feridas operatórias nas cesarianas realizadas em uma maternidade, no período de 2015 a 2018
}

\author{
Analysis of the use of antibiotics in the prophylaxis of operating wounds in cesarians \\ carried out in a maternity, from 2015 to 2018
}
Análisis del uso de antibióticos en la profilaxis de heridas operativas en cesarios realizadas en un hospital de maternidad, de 2015 a 2018

Iran Grijó Praia ${ }^{1,2}$, Sirlene Maria da Silva ${ }^{1 *}$.

\begin{abstract}
RESUMO
Objetivo: Analisar o uso de antibióticos na profilaxia de ferida operatória, através da demonstração dos esquemas de antibióticos prescritos no pré e pós-operatório, análise do perfil epidemiológico e fatores de risco para infecção do sítio cirúrgico (ISC) nas cesarianas realizadas em uma maternidade. Métodos: Estudo transversal, caráter retrospectivo e abordagem quantitativa, a partir da análise de prontuários das pacientes submetidas a cesarianas e que tiveram ISC, no período de 2015 a 2018. Resultados: A amostra foi 303 gestantes. A média de idade foi 24,1 anos $(24,8 \%)$, a indicação mais prevalente para cesariana foi Préeclâmpsia (17,2\%). Os principais critérios clínicos de ISC foram eritema $(38,9 \%)$ e edema (36,6\%). A antibioticoprofilaxia foi administrada em $87 \%$ das pacientes, sendo realizada com cefalosporina de primeira geração. O esquema mais utilizado para tratamento da ISC foi o esquema tríplice Cefalotina associada a Gentamicina e Metronidazol (75\%). Conclusão: As taxas de ISC foram elevadas durante o período de estudo. Antibioticoprofilaxia não define proteção absoluta contra infecção de ferida operatória. A ISC prolonga em torno de 7 a 15 dias o tempo de internação e reinternação das pacientes que possuem infecção do sítio cirúrgico tardio.
\end{abstract}

Palavras-chave: Cesárea, Infecção da ferida cirúrgica, Infecção puerperal.

\begin{abstract}
Objective: To analyze the use of antibiotics in prophylaxis of surgical wounds, by demonstrating the antibiotic regimens prescribed in the pre and postoperative periods, analysis of the epidemiological profile and risk factors for surgical site infection (SSI) in cesarean sections performed in a maternity. Methods: Crosssectional study, retrospective and quantitative approach, based on the analysis of medical records of patients who underwent cesarean sections and who had SSI, from 2015 to 2018. Results: The sample was 303 pregnant women. The mean age was 24.1 years $(24.8 \%)$, the most prevalent indication for cesarean section was Pre-eclampsia (17.2\%). The main clinical criteria for SSI were erythema (38.9\%) and edema (.6\%). Antibiotic prophylaxis was administered to $87 \%$ of the patients, with first-generation cephalosporin. The most used regimen for treating $36 \mathrm{SSI}$ was a triple Cephalothin regimen associated with Gentamycin and Metronidazole (75\%). Conclusion: SSI rates were high during the study period. Antibiotic prophylaxis does not define absolute protection against wound infection. SSI prolongs the length of hospitalization and readmission of patients who have late surgical site infection by 7 to 15 days.
\end{abstract}

Keywords: Cesarean section, Surgical wound infection, Puerperal infection.

\footnotetext{
1 Universidade Estadual do Amazonas, Manaus - AM. *E-mail: sirlenemariadasilva315@gmail.com

2 Fundação de Medicina Tropical, Manaus - AM.
}

SUBMETIDO EM: 1/2021

ACEITO EM: 2/2021

PUBLICADO EM: 2/2021 


\section{RESUMEN}

Objetivo: Analizar el uso de antibióticos en la profilaxis de heridas quirúrgicas, mediante la demostración de los regímenes antibióticos prescritos en el pre y posoperatorio, análisis del perfil epidemiológico y factores de riesgo de infección del sitio quirúrgico (ISQ) en cesáreas realizadas en un hospital de maternidad. Métodos: Estudio transversal, de abordaje retrospectivo y cuantitativo, basado en el análisis de historias clínicas de pacientes que fueron sometidas a cesáreas y que tuvieron ISQ, de 2015 a 2018. Resultados: La muestra fue de 303 mujeres embarazadas. La edad media fue de 24,1 años (24,8\%), la indicación de cesárea más prevalente fue la Preeclampsia (17,2\%). Los principales criterios clínicos de ISQ fueron eritema (38,9\%) y edema $(36,6 \%)$. Se administró profilaxis antibiótica al $87 \%$ de los pacientes, con cefalosporina de primera generación. El régimen más utilizado para tratar la ISQ fue un régimen triple de cefalotina asociado con gentamicina y metronidazol (75\%). Conclusión: Las tasas de ISC fueron altas durante el período de estudio. La profilaxis con antibióticos no define una protección absoluta contra la infección de la herida. ISC prolonga el tiempo de hospitalización y rehospitalización de los pacientes que tienen infección tardía del sitio quirúrgico de 7 a 15 días.

Palabras clave: Cesárea, Infección de la herida quirúrgica, Infección puerperal.

\section{INTRODUÇÃO}

Não há dúvidas na literatura sobre a elevada importância do parto cesáreo para ajudar a salvar vidas e prevenir sequelas neonatais, como as originadas de partos vaginais distócicos. Por outro lado, o aumento da incidência da cesariana aumenta a morbidade e o custo operacional para o sistema, podendo tornar-se um problema potencial (FREITAS F, et al., 2001).

O Brasil vem apresentando, nos últimos anos, uma das mais elevadas taxas de cesáreas do mundo, correspondendo a $55 \%$ do total de partos, enquanto a média recomendada é de 10 a $15 \%$, mesmo frente a atitude do Ministério da Saúde que vem buscando alterar o modelo de assistência ao parto considerado hoje como intervencionista e caracterizado por vezes como evento cirúrgico. O parto cesáreo constitui uma importante questão na assistência à saúde da mulher em função das possíveis complicações e pela maior morbimortalidade associada quando comparado ao parto normal (ROMANELLI RMC, 2014; ZIMMERMMANN JB, 2009).

A infecção no período puerperal é um problema grave para a saúde pública, confirmado pelas altas taxas de morbi-mortalidade na população em geral. Ademais, o próprio parto cesáreo constitui um dos principais riscos para a infecção do sítio cirúrgico, ao passo que este permite a entrada de bactérias devido ao rompimento da barreira cutânea de proteção (RICCI SS, 2013).

O parto cesárea favorece as complicações puerperais, sendo fator predisponente para elevar o risco de endometrite, bacteremia, abscesso ou tromboflebite pélvica e morte por infecção. Dentre essas complicações possíveis no período puerperal, as infecciosas são as mais comuns sendo que a infecção de sítio cirúrgico é de maior ocorrência (CHIANCA LM, 2015). Nesse sentido, a infecção do sítio cirúrgico enquadra-se como uma das principais infecções relacionadas à assistência à saúde no Brasil, caracterizando-se por ocorrer nos primeiros 30 dias após o procedimento cirúrgico (ANVISA, 2017).

A infecção do sítio cirúrgico pós-cesárea pode ser conceituada como um processo infeccioso inflamatório da ferida ou cavidade operada com drenagem de secreção purulenta, com ou sem cultura positiva. A infecção pode ser restringida à região de incisão operatória, e neste caso a área apresentará sinais flogísticos como edema e hiperemia, ou pode ainda abranger tecidos que foram manuseados durante a cirurgia adjacentes à região de incisão (MARTINS ACM e SILVA LKM, 2006).

Os fatores de risco relacionados à infecção do sítio cirúrgico dividem-se em duas categorias: origem exógena (contaminação externa, relacionada ao procedimento propriamente dito e condições locais de higiene) ou endógena, relacionada à própria flora genital da paciente. Os fatores endógenos, tais como a flora 
materna (do endométrio, líquido amniótico e cervicovaginal), causam a maioria das ISC pós-cesárea (ROMANELLI RMC, et al., 2014; ANVISA, 2017).

É de extrema importância ressaltar que alguns fatores, além da técnica operatória propriamente dita, são importantes como proteção de fontes exógenas como, por exemplo, o banho no pré-operatório, tricotomia apenas na sala de operações, antissepsia do local da incisão, realização da antibioticoprofilaxia na indução anestésica, degermação e paramentação de toda a equipe cirúrgica, proteção da ferida operatória com curativo estéril por $24 \mathrm{~h}$ e controle de pessoas circulantes na sala de operações (LACERDA R, 2003).

Entretanto, após $24 \mathrm{~h}$ do procedimento, a ferida cirúrgica está selada e, portanto, protegida da contaminação exógena. As infecções à distância podem ser fonte de microrganismos que contaminam a ferida cirúrgica e devem ser pesquisadas e tratadas no pré-operatório. Por isso, há necessidade de avaliação préoperatória de todo paciente cirúrgico (SANTOS AA, et al., 2005).

As Infecções do Sítio Cirúrgico (ISC) são as complicações operatórias mais relevantes, levando-se em consideração as frequentes subnotificações dos casos, além da falta de vigilância ativa pós-alta, alta antes do tempo previsto e atraso da paciente para conseguir ser atendida quando considerada a contra-referência para as Unidades Básicas de Saúde (UBS) (CUNHA MR, et al., 2018).

O objetivo deste estudo é analisar o uso de antibióticos na profilaxia das feridas operatórias nas cesarianas realizadas em uma maternidade, no período de 2015 a 2018, de forma a demonstrar os esquemas de antibióticos prescritos na antibioticoprofilaxia e no tratamento das feridas operatórias e identificar os fatores epidemiológicos e socioeconômicos para esta patologia. Diante da relevância de investigar infecções puerperais, especialmente as relacionadas com o parto cesáreo, a realização desse estudo se justifica pelas repercussões negativas na recuperação da mulher no período pós-parto, comprometendo a involução puerperal satisfatória, prolongando o tempo de hospitalização e retardando o vínculo mãe/recém-nascido e família.

\section{MÉTODOS}

A presente proposta de estudo consiste em uma pesquisa transversal, de caráter retrospectivo e abordagem quantitativa, a partir da análise de prontuários das pacientes que realizaram cesarianas e tiveram infecção de feridas operatórias, em uma maternidade, no período de 2015 a 2018. A população do presente estudo trata-se de mulheres que realizaram partos do tipo cesáreo e que apresentaram infecção de ferida pós-operatória, no período de 2015 a 2018 em uma maternidade de Manaus. O tamanho amostral da pesquisa foi de 167 pacientes.

Durante a coleta, foram catalogados os dados referentes à idade, escolaridade, renda familiar, indicação da realização da cesariana, antibioticoprofilaxia prescrita para a cirurgia, antibioticoterapia prescrita para a infecção da ferida cirúrgica e tempo de tratamento. Estes dados foram coletados através de uma ficha de coleta.

Os critérios de inclusão do estudo foram mulheres que foram submetidas a parto cesáreo e mulheres que apresentaram infecção de feridas operatórias na maternidade em estudo. O critério de exclusão adotado foram prontuários sem dados acerca da antibioticoprofilaxia ou antibioticoterapia. O projeto foi submetido e aprovado pelo Comitê de Ética em Pesquisa (CEP) através da Plataforma Brasil, com o CAEE 11931019.8.0000.5016.

\section{RESULTADOS}

As taxas de realização de cesarianas nos anos de 2015, 2016, 2017 e 2018 na maternidade em estudo foram respectivamente: 40,7\% ( $n=3352) ; 39,4 \%$ ( $n=3387) ; 41,6 \% \quad(n=3414)$ e $42 \%(n=3445)$, como mostra o Gráfico 1 a seguir. Quanto à taxa global de infecção de sítio cirúrgico, nesses 4 anos, foi de 3,83\%, uma vez que do total de 13.498 partos cesáreos, 518 evoluíram com Infecção do Sítio Cirúrgico. 
Gráfico 1 - Número de cesáreas realizadas por ano na instituição ( $\mathrm{N}$ total = 13.598).

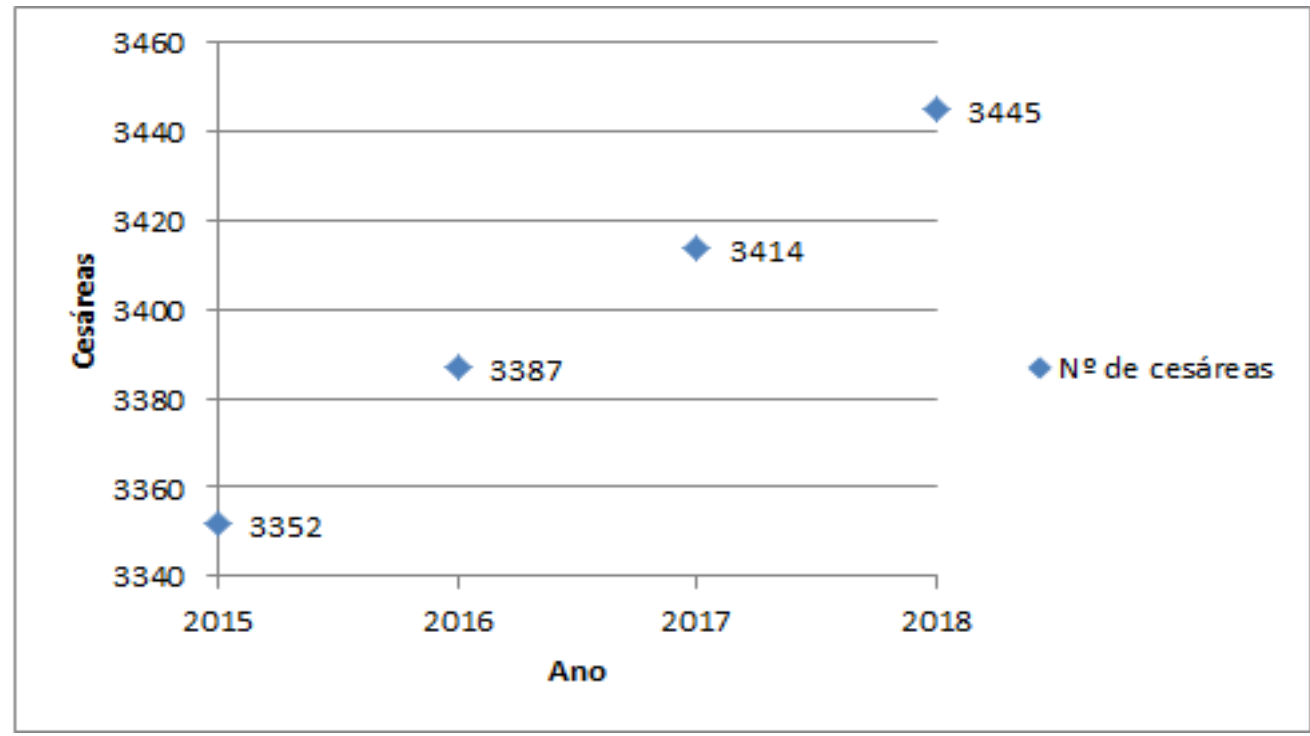

Fonte: Praia IG e Silva SM, 2021.

Foram investigados 518 casos de infecção de sítio cirúrgico, de um total de 13.498 cesarianas. A amostra deste estudo foi de 303 gestantes submetidas a partos cesáreos com Infecção de Sítio Cirúrgico (ISC) no período de janeiro de 2015 a dezembro de 2018 que se adequaram aos critérios de inclusão. Os 215 prontuários restantes não contemplavam os dados requeridos pelo projeto, sendo, pois, excluídos das análises. Os 303 prontuários disponíveis para análise indicaram que a média de idade do grupo de pacientes foi de 24,7 anos. A maioria das pacientes $(24,8 \%, n=75)$ estão no intervalo de idade entre 18 e 21 anos. Apenas 9 pacientes (3\%) tinham mais de 41 anos, e 46 pacientes possuíam abaixo de 18 anos, conforme 0 Gráfico 2.

Gráfico 2 - Faixa etária das pacientes que tiveram infecção de sítio cirúrgico após cesárea ( $\mathrm{n}$ total $=303$ ).

80

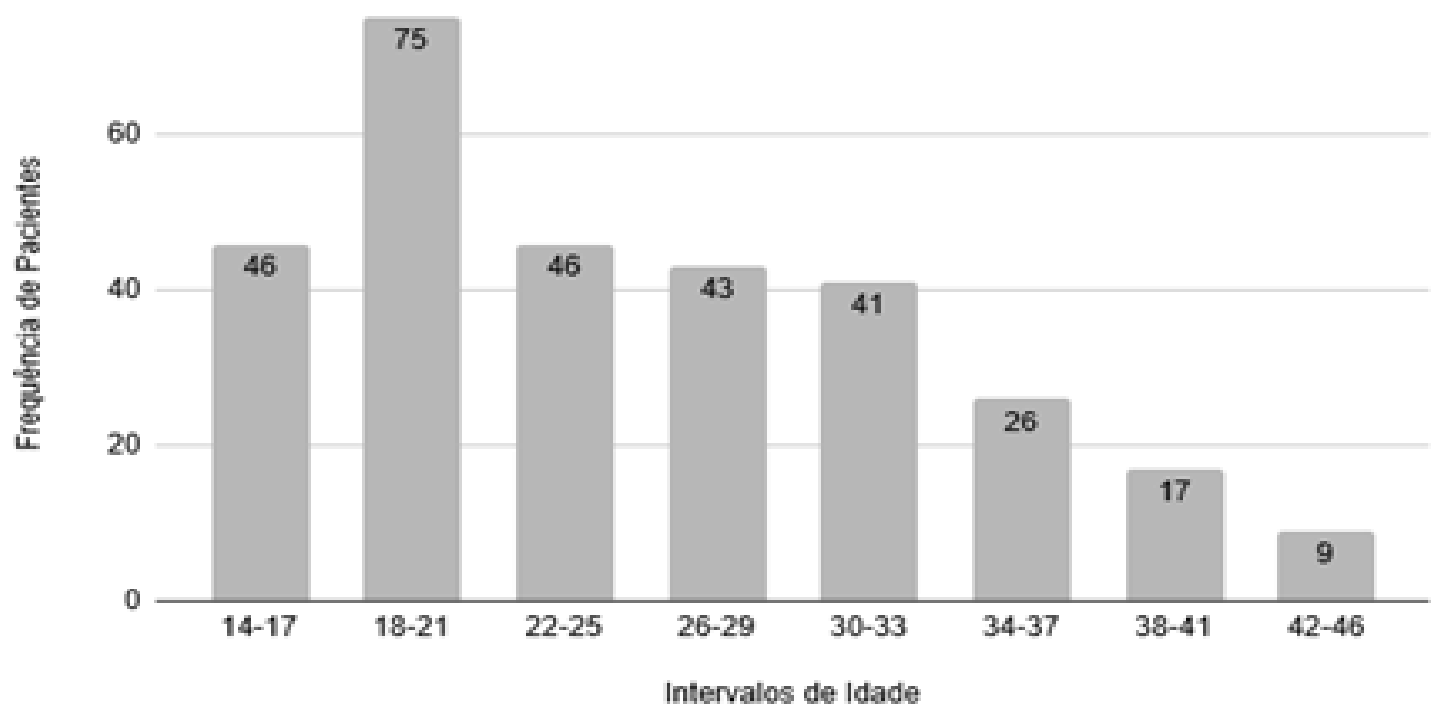

Fonte: Praia IG e Silva SM, 2021.

Como descrito na Tabela 1, em 33,3\% dos casos $(n=101)$, as pacientes foram reinternadas devido à infecção do sítio cirúrgico. Em relação à paridade, $37 \%(n=112)$ apresentaram infecção do sítio cirúrgico na primeira gestação, secundigesta $24,1 \%(n=73)$, seguida das multíparas com $38.9 \%(n=118)$. 
Ao analisar-se as condições socioeconômicas podemos observar que o baixo índice de escolaridade associado à baixa renda contribuem para elevar o número de casos de infecção de ferida operatória. Analfabetismo e ensino fundamental totalizam $60,39 \%(n=183)$ e a baixa renda abaixo de 3 salários mínimos $87,46 \%(n=265)$. As comorbidades associadas às pacientes, notou-se que o mais prevalente foi a Síndrome Hipertensiva, presente em 17,2\% $(n=52)$ das pacientes, seguida de Infecção do Trato Urinário, presente em $15,5 \%(n=47)$ das mesmas.

Em relação ao tempo de hospitalização, em torno de 10 dias, em uso de antibioticoterapia em esquema tríplice (Cefalosporina de terceira geração associada à Gentamicina e Metronidazol) de Acordo com a Comissão de Controle de Infecção Hospitalar $(\mathrm{CCIH})$ da maternidade onde foi realizado o estudo, caso a paciente não evolua satisfatoriamente, com melhora do quadro de hiperemia, hematoma de parede e exsudação purulenta de ferida operatória é realizado a cultura da secreção com antibiograma, com o objetivo de instituir um tratamento individualizado, baseado na resistência bacteriana que provavelmente já está instalada, aumentando o tempo de internação para em torno de 20 a 25 dias.

Em relação ao pré-natal, $62(n=188)$ das pacientes o realizaram, independentemente do número de consultas. No entanto, $10,6 \%$ ( $n=32)$ das pacientes não tinham essa informação no prontuário e $27,4 \%$ ( $n=83)$ não realizaram o pré-natal.

Tabela 1 - Características das pacientes que tiveram infecção de sítio cirúrgico após cesárea na maternidade, 2015-2018 ( $\mathrm{N}$ total = 303).

\begin{tabular}{|c|c|c|}
\hline Variáveis & $\mathbf{N}$ & $\%$ \\
\hline Total de prontuários analisados & 303 & 100 \\
\hline Reinternação & 101 & 33,3 \\
\hline \multicolumn{3}{|l|}{ Paridade } \\
\hline Primípara & 112 & 37 \\
\hline Secundigesta & 73 & 24,1 \\
\hline Multípara & 118 & 38,9 \\
\hline \multicolumn{3}{|l|}{ Escolaridade } \\
\hline Analfabeta & 25 & 8,25 \\
\hline Ensino Fundamental & 158 & 52,14 \\
\hline Ensino Médio & 83 & 27,39 \\
\hline Ensino Superior & 37 & 12,22 \\
\hline \multicolumn{3}{|l|}{ Renda Familiar } \\
\hline < de 1 salário mínimo & 158 & 52,14 \\
\hline De 1 a 3 salário mínimos & 107 & 35,32 \\
\hline > 3 salário mínimos & 38 & 12,54 \\
\hline \multicolumn{3}{|l|}{ Comorbidades } \\
\hline Diabetes Mellitus & 13 & 4,3 \\
\hline Infecção do Trato Urinário & 47 & 15,5 \\
\hline Infecção por HIV & 7 & 2,3 \\
\hline Sífilis & 11 & 3,6 \\
\hline Síndrome Hipertensiva & 52 & 17,2 \\
\hline \multicolumn{3}{|l|}{ Tempo de Hospitalização } \\
\hline $1-5$ dias & 17 & 5,6 \\
\hline 6-10 dias & 88 & 29 \\
\hline $11-15$ dias & 69 & 22,8 \\
\hline $16-20$ dias & 88 & 29 \\
\hline $21-25$ dias & 29 & 9,6 \\
\hline 26-30 dias & 6 & 2 \\
\hline$>30$ dias & 6 & 2 \\
\hline \multicolumn{3}{|l|}{ Pré-natal } \\
\hline Sim & 188 & 62 \\
\hline Não & 83 & 27,4 \\
\hline Não Informado & 32 & 10,6 \\
\hline
\end{tabular}

Fonte: Praia IG e Silva SM, 2021. 
Dentre as pacientes que realizaram pré-natal, apenas $5,9 \%(n=11)$ das pacientes realizaram 1 consulta, 9,5\% ( $n=18) 2$ consultas, $11,7 \%(n=22) 3$ consultas, 8,5\% $(n=16) 4$ consultas, $11,7 \%(n=22) 5$ consultas e $52,7 \%$ ( $n=99)$ tiveram seis ou mais consultas. A média de consultas no pré-natal foi de 5,72 .

As indicações clínica e obstétricas que levaram à realização da cesárea mais frequentes foram a Préeclâmpsia, com $17,2 \%$ dos casos ( $n=52$ ); seguida da Desproporção Cefalopélvica, que predominou em 12,2\% dos casos ( $n=37$ ) e Amniorrex prematura com $9,2 \%$ dos casos $(n=28)$.

Os principais critérios clínicos associados à infecção do sítio cirúrgico estão apresentados no Gráfico 3. O sinal mais prevalente foi o eritema, presente em $38,9 \%$ dos casos $(n=118)$, seguido pelo edema, relatado em $36,6 \%(n=111)$. Outros critérios clínicos frequentes foram a febre, em $20,1 \%(n=61)$ e a deiscência da sutura, em $15,5 \%$ dos casos $(n=47)$.

Gráfico 3 - Frequência de critérios clínicos em infecções de ferida cirúrgica ( $N$ total $=388$ ).

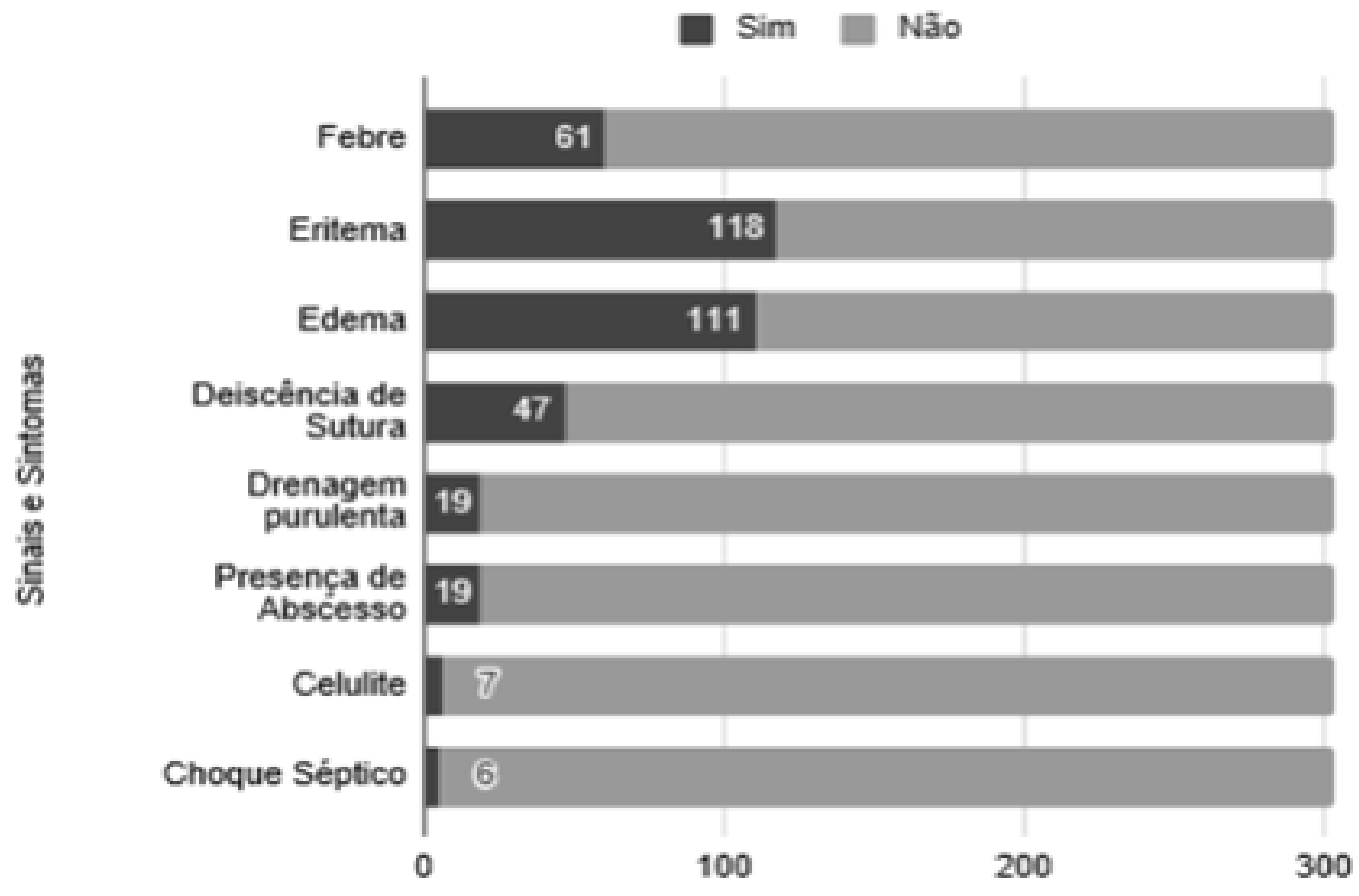

Fonte: Praia IG e Silva SM, 2021.

Pode-se observar através da Tabela 2 que a antibioticoprofilaxia foi realizada em 97,35\% $(n=295)$ das pacientes que apresentaram posteriormente ferida operatória, sendo 2,65\% $(n=8)$ não realizaram antibioticoprofilaxia. A classe utilizada na profilaxia foi a cefalosporina de primeira geração, dentro da qual, o esquema mais utilizado foi a Cefalotina, representando $54,12 \%(n=164)$ dos casos, seguido da Cefazolina, em 45,88\% ( $n=131)$ dos casos, administrada na indução anestésica.

Tabela 2 - Perfil da antibioticoprofilaxia utilizada nas pacientes que evoluíram para infecção de sítio cirúrgico após cesárea ( $\mathrm{N}$ total $=303$ ).

\begin{tabular}{lcc}
\hline Variáveis & $\mathbf{N}$ & $\%$ \\
\hline Antibioticoprofilaxia & & \\
\hline Sim & 295 & 97,35 \\
Não realizado & 8 & 2,65 \\
\hline Total & 303 & $100 \%$ \\
\hline Antibiótico de escolha para antibioticoprofilaxia & & \\
\hline Cefalotina & 164 & 55,60 \\
Cefazolina & 131 & 44,4 \\
\hline Total & 295 & $100 \%$ \\
\hline Fonte: Praia & &
\end{tabular}


Pode-se observar na Tabela 3 quais os antibióticos mais utilizados no tratamento da ferida operatória infectada, além de quantas vezes ocorreu falha terapêutica (necessidade de substituição). O esquema mais utilizado foi a Cefalotina associada a Gentamicina e Metronidazol, em $65 \%$ dos casos $(n=197)$, com tempo médio de terapia de 8,38 dias, e com falha terapêutica em 29 casos.

O segundo esquema mais utilizado foi a Ceftriaxona associada a Gentamicina e Metronidazol, em 14,8\% dos casos $(n=45)$, com tempo médio de terapia de 8,54 dias, e com falha terapêutica em 2 casos. $O$ terceiro esquema mais utilizado foi a Cefalotina em monoterapia, em $4,2 \%$ dos casos ( $n=13$ ), com tempo médio de terapia de 5,8 dias, e com falha terapêutica em 4 casos.

Tabela 3 - Perfil do uso de antibióticos de $1^{\circ}$ escolha no tratamento de infecção de sítio cirúrgico após cesárea $(\mathrm{N}$ total $=303)$.

\begin{tabular}{ccccc}
\hline Antibióticos & $\begin{array}{c}\text { Frequência de } \\
\text { escolha (N) }\end{array}$ & $\%$ & $\begin{array}{c}\text { Tempo médio de uso } \\
\text { (dias) }\end{array}$ & $\begin{array}{c}\text { Quantas vezes foi } \\
\text { necessário } \\
\text { substituir esquema }\end{array}$ \\
\hline $\begin{array}{c}\text { Cefalotina }+ \\
\text { Gentamicina }+ \\
\text { Metronidazol }\end{array}$ & 197 & 65,0 & 8,38 & 29 \\
\hline $\begin{array}{c}\text { Ceftriaxona }+ \\
\text { Gentamicina }+ \\
\text { Metronidazol }\end{array}$ & 45 & 14,85 & 8,54 & 2 \\
\hline Cefalotina & 13 & 4,30 & 5,8 & 4 \\
\hline $\begin{array}{c}\text { Clindamicina }+ \\
\text { Gentamicina }\end{array}$ & 9 & 2,98 & 8,5 & 0 \\
\hline $\begin{array}{c}\text { Cefalotina }+ \\
\text { Clindamicina }+ \\
\text { Gentamicina }\end{array}$ & 5 & 1,65 & 9 & 1 \\
\hline Ceftriaxona & 5 & 1,65 & 8 & 0 \\
\hline $\begin{array}{c}\text { Ceftriaxona }+ \\
\text { Clindamicina }\end{array}$ & 5 & 1,65 & 7,5 & 0 \\
\hline Outros $(\mathrm{n}<5)$ & 24 & 7,92 & 9,3 & 4 \\
\hline Fonte: Praia IG 6 & & & \\
\hline
\end{tabular}

Fonte: Praia IG e Silva SM, 2021.

O antibiótico mais utilizado como segunda escolha foi a Ceftriaxona, escolhida em $50 \%$ dos casos $(n=20)$. Em segundo lugar, encontra-se o esquema Cefalotina associada a Gentamicina e Metronidazol, com uma porcentagem de $27,5 \%(n=11)$. Em terceiro lugar, encontram-se a Ceftriaxona associada Clindamicina $12,5 \%$ dos casos $(n=5)$ e a Clindamicina $10 \%$ dos casos $(n=4)$ em monoterapia.

A ultrassonografia abdominal foi realizada em $47,85 \%(n=145)$ das pacientes. Dessas $52,4 \%(n=76)$ destes exames evidenciaram abscesso de parede e 46,3\% ( $n=67)$ hematoma, seguidos pela combinação de hematoma e abscesso representando $0,68 \%,(n=1)$ e $0,68(n=1)$ sem alterações. Sobre a drenagem abdominal, 49,65\% $(n=72)$ das puérperas foram submetidas a este procedimento, sendo que na maioria $(56,7 \%, n=38)$ drenou-se hematoma e o restante, $44,73 \%(n=34)$, abscesso. Por fim, o procedimento de ressutura foi realizado em menos da metade das pacientes, isto é, $43,44 \%(n=63)$.

\section{DISCUSSÃO}

O Brasil apresenta a segunda maior taxa de cesáreas do mundo com $55 \%$, ficando atrás da República Dominicana, onde a taxa é de $56 \%$ (WHO, 2018). No local de estudo, a taxa média de cesárea entre os anos de 2015 e 2018 foi de 40,9\%, abaixo dos índices brasileiros, mas muito além do preconizado pela OMS. Quanto à taxa média de ISC, nesses 4 anos, foi de 3,83\%, muito acima do valor de $2,8 \%$ encontrado no estudo do Hospital de Clínicas de Porto Alegre (BENINCASA BC, 2012).

A idade materna acima de 35 anos é considerada como fator de risco para infecção de sítio cirúrgico póscesariana (CAPUZZI IF, et al., 2007; PAIVA VP, et al., 2012). Neste estudo, 13,9\% $(n=42)$ das pacientes tem 
mais de 35 anos. A média de idade foi de 24,7 e a maioria das pacientes $(24,8 \%, n=75)$ estão no intervalo de idade entre 18 e 21 anos. O fator idade não foi uma agravante para infecção de ferida operatória.

Sobre a paridade, a maioria das pacientes eram primíparas totalizando $37 \%(n=112)$ de todas as pacientes analisadas, seguidas das multíparas com 33,6\% $(n=102)$. É apontado a primiparidade como fator de risco para infecção puerperal (MAHARAJ D, 2007). Em um estudo foram encontrados como fatores predisponentes à ISC pós-cesárea: a anemia, a HAS, infecção puerperal em cesárea prévia e o tabagismo (MEDEIROS GO e SOUZA LM, 2010). O que se corrobora de forma parcial, ao que foi encontrado neste estudo, no qual o mais incidente foi a síndrome hipertensiva com $17,2 \%$, seguida de infecção do trato urinário com $15,5 \%$, estando também identificados em menor número o diabetes mellitus e as infecções por sífilis e HIV.

Sobre o pré-natal, observou-se que $62 \%(n=188)$ das pacientes do estudo realizaram. O número de consultas de pré-natal deve ser no mínimo seis para que o acompanhamento seja considerado adequado (PETTER CE, 2013). Em nossa amostra das pacientes que realizaram pré-natal, somente 52,7\% ( $N=99$ ) tiveram seis consultas ou mais e a média de consultas no pré-natal foi de 5,72. É importante mencionar que $27,4 \%(N=83)$ não realizaram o pré-natal. O restante, $10,6 \%(n=32)$, não havia informação acerca deste tópico no prontuário. $\mathrm{O}$ que se mostra mais um fator predisponente para infecção de ferida operatória em nosso estudo.

O aumento no tempo de hospitalização interfere no puerpério da mulher com seu recém-nascido e, ainda promove a ocupação dos leitos por mais tempo, impedindo a disponibilidade de vagas para outros pacientes. Nesta pesquisa, a maioria das puérperas permaneceu internada em um período superior a 10 dias, sugerindo que o tratamento adotado para estas não obteve resultado rápido e eficiente (SOUSA AFL, et al., 2020).

O número de gestantes que iniciam o pré-natal é um dos parâmetros utilizados para avaliar a qualidade da assistência materno-fetal. O pré-natal tem papel fundamental em termos de prevenção e/ou detecção precoce de patologias, tanto maternas como fetais (FEBRASGO, 2014).

As principais indicações para cesariana foram: pré-eclâmpsia, desproporção cefalopélvica e amniorrexe prematura. Considerando esses últimos dados, verifica-se que os resultados corroboraram com os encontrados em outros estudos (SCHNEID-KOFMAN N, 2005; BENINCASA BC, 2012). O primeiro identificou como fatores predisponentes diabetes mellitus, hipertensão e ruptura prematura de membranas, já o segundo demonstrou que amniorrexe prematura é predisponente de riscos.

Existem evidências consistentes de que a antibioticoprofilaxia administrada antes da incisão cutânea nas cesarianas diminui a incidência de infecção de sítio cirúrgico (OWENS SM, 2009; CONSTANTINE MM, 2008). Desta forma, a maior parte das pacientes, isto é, $57 \%$ (173) realizaram a antibioticoprofilaxia no momento correto (pré-operatório). O restante, $43 \%$, não realizou a antibioticoprofilaxia.

A profilaxia da cesariana é indicada após ruptura da membrana amniótica há mais de 6 horas, ou quando o trabalho de parto tem mais de 12 horas e nas situações gerais de risco. O esquema recomendado é Cefalotina ou Cefazolina 1 a 2 g, IV, após o clampeamento do cordão em dose única (FRONZO C, 2019; LACERDA R, 2003).

De acordo com um estudo de meta-análise, não há diferença de efetividade entre as cefalosporinas de primeira, segunda e terceira geração na profilaxia de infecção de ferida operatória, sendo ambos altamente eficazes (HOPKINS L e SMAIL F, 1999). Desta forma, todos os antibióticos utilizados no pré-operatório, ou seja, Cefalotina e Cefazolina apresentam, de acordo com a literatura, capacidade de evitar a infecção de sítio cirúrgico. No entanto, foi relatado que a gravidez é capaz de interferir na farmacocinética da ceftriaxona e da gentamicina, tornando-as menos eficazes (POPOVIC J, 2007).

Os achados neste estudo demonstraram que a infecção de ferida operatória ocorreu mesmo adotando o tempo de administração e o tipo de antibiótico. Por isso, outros fatores que influenciam a eficiência da antibioticoprofilaxia que não estavam informados no prontuário, como tempo de cirurgia, preparo da paciente com banho, degermação, técnica cirúrgica, tempo cirúrgico, experiência cirúrgica e tipo de curativo utilizado deveriam ser analisados para ser encontrada a falha na profilaxia realizada. 
Na terapêutica das complicações cirúrgicas o esquema mais utilizado foi a Cefalotina associada a Gentamicina e Metronidazol, em $65 \%$ dos casos. Em um estudo, foram analisadas culturas obtidas de foco de infecção puerperal, e demonstrou-se que a flora presente é polimicrobiana, sendo mais prevalente a anaeróbia. Em material colhido durante as cesáreas encontraram-se microorganismos anaeróbios e aeróbios em 63,0\%, anaeróbios em 30,0\% 26 e aeróbios em apenas 7,0\% (SANTOS VB, 2017).

A associação da Cefalotina aos antibióticos supracitados no tratamento das infecções puerperais nas pacientes que já haviam realizado antibioticoprofilaxia com Cefalosporina de primeira geração, mesmo sendo uma ótima opção no tratamento de uma gama de infecções, inclusive em infecções de pele e da estrutura da pele, é desnecessária, já que após falha da antibioticoprofilaxia com cefalosporinas deve-se optar por classes diferentes de antibióticos, além de sua utilização excessiva ser considerada um dos fatores que mais contribui para o problema da resistência microbiana (LOUREIRO RJ, 2016).

A identificação do microrganismo causador da infecção do sítio cirúrgico é necessária, pois orientará no sentido da prescrição de antibioticoterapia dirigida e fornecerá dados para análise da flora prevalente numa dada instituição (SANTOS VB, 2017). No presente estudo, foi observado que nenhuma paciente fez coleta de fragmento da ferida operatória para cultura. Ou seja, todas as pacientes internadas para o tratamento da ISC pós-cesárea iniciaram algum tipo de antibioticoterapia sem análise do agente etiológico. Somente se a paciente não apresentasse uma evolução satisfatória, seria realizado a cultura com antibiograma, para conduzir individualmente, os casos que houvesse falhas a antibioticoterapia empírica, pela inviabilidade do exame foram realizadas em torno de 10 culturas por ano, onde não tivemos acesso, que conforme o relato do responsável pela $\mathrm{CCIH}$ se extraviou.

\section{CONCLUSÃO}

A taxa de infecção de sítio cirúrgico foi elevada durante o período de estudo (3,83\%). Os extremos de idade e pacientes com comorbidades são os mais propensos a desenvolver infecção de ferida pós-operatória. Pacientes com baixo índice socioeconômico possuem maior risco de apresentarem infecção de ferida operatória. A antibioticoprofilaxia não define proteção absoluta contra a infecção de ferida operatória. As classes de antibióticos mais utilizadas para o tratamento da ferida operatória infectada são as cefalosporinas de primeira geração, associadas ou não com aminoglicosídeos e metronidazol. A importância de estudos sobre infecção puerperal reside no fato de constituir-se em uma das principais causas de morbimortalidade no período puerperal.

\section{REFERÊNCIAS}

1. ANVISA. Caderno 2 - Critérios Diagnósticos de Infecção Relacionada à Assistência à Saúde. 2017. Disponível: www.anvisa.gov.br/segurancadopaciente. Acessado em: 20 de julho de 2020.

2. ANVISA. Diagnóstico do Controle de Infecção Hospitalar no Brasil. 2005. Disponível: www.anvisa.gov.br. Acessado em: 18 de julho de 2020.

3. ARAÚJO ABS, et al. Ocorrência de infecções de sítio cirúrgico pós-cesárea em uma maternidade pública. Revista Enfermería Actual de Costa Rica, 2019; 37: 16-29.

4. BENINCASA BC, et al. Taxas de Infecção relacionados a partos cesáreos e normais no hospital e Clínicas de Porto Alegre. Revista HCPA, 2012; 32: 5-9.

5. CAPUZZI IF, et al. Análise dos fatores de risco em puérperas com infecção de sítio cirúrgico em unidade hospitalar de obstetrícia. Revista Perspect Med, 2007; 18: 11-6.

6. CHIANCA LM, et al. Índice de risco cirúrgico e infecção de ferida operatória em puérperas submetidas a cesarianas. Revista de Epidemiologia e Controle de Infecções, 2015; 5: 17-22.

7. CONSTANTINE MM, et al. Timing of perioperative antibiotics for cesarean delivery: a metaanalysis. Revista American Journal of Obstetrics and Gynecology, 2008; 199: 1-6.

8. CUNHA MR, et al. Identificação da infecção do sítio cirúrgico pós-cesariana: consulta de enfermagem. Revista Brasileira de Enfermagem, 2018; 71: 1395-1403.

9. FEBRASGO. Manual de assistência pré-natal. 2014. Disponível em: portaldeboaspraticas.iff.fiocruz.br/biblioteca. Acessado em: 14 de julho de 2020.

10. FREITAS F, et al. Rotinas em Obstetrícia. 4 ed. Porto Alegre: Artmed, 2001; 321p.

11. FRONZO C. Infecciones quirúrgicas postparto: prevención, monitoreo y uso de apósitos con cloruro de dialquilcarbamilo (DACC). Revista Journal of Wound Care, 2019; 29: 1-2. 
12. HOPKINS L, SMAIL F. Antibiotic prophylaxis regimens and drugs for cesarean section. Revista Cochrane Database of Systematic Reviews, 1999; 2: 11-36.

13. LACERDA R. Controle de infecção em centro cirúrgico: fatos, mitos e controvérsias. Rio de Janeiro: Atheneu, 2003; $134 p$.

14. LOUREIRO RJ, et al. O uso de antibióticos e as resistências bacterianas: breves notas sobre a sua evolução. Revista Portuguesa de Saúde Pública, 2016; 34: 1-2.

15. MAHARAJ D. Puerperal pyrexia: a review. Part II. Revista Obstetrical \& Gynecological Survey, 2007; 62: 400-406.

16. MARTINS ACM, SILVA LK. Revisões sistemáticas de antibioticoprofilaxia em cesarianas. Revista Cadernos de Saúde Pública, 2006; 22: 101-107.

17. MEDEIROS GO, SOUZA LM. Proposta de criação de protocolo de enfermagem para o cuidado de pacientes com abscesso de parede pós-cesária. Revista Comunicação em Ciências da Saúde, 2010; 21: 09-20.

18. OMS. Intrapartum care for a positive childbirth experience. 2018. Disponível em: www.who.int/reproductivehealth. Acessado em: 05 de julho de 2020.

19. OWENS SM, et al. Antimicrobial prophylaxis for cesarean delivery before skin incision. Revista Obstetrics and Gynecology, 2009; 114: 573-9.

20. PAIVA VP, et al. Obesidade materna em gestações de alto risco e complicações infecciosas no puerpério. Revista da Associação Médica Brasileira, 2012; 58: 4-9.

21. PETTER CE, et al. Fatores relacionados a infecções de sítio cirúrgico após procedimentos obstétricos. Revista Scientia Medica, 2013; 23: 28-33.

22. POPOVIC J, et al. Influence of pregnancy on ceftriaxone, cefazolin and gentamicin pharmacokinetics in caesarean vs. non-pregnant sectioned women. Revista Journal of Clinical Pharmacy and Therapeutics, 2007; 32: 595-602.

23. RICCI SS. Enfermagem materno-neonatal e saúde da mulher. ํㅜㄹ. Rio de Janeiro: Guanabara Koogan, 2013; 193p.

24. ROMANELLI RMC, et al. Fatores de risco para infecção de ferida cirúrgica em puérperas submetidas a cesarianas em Hospital Universitário de referência. Revista Epidemiol Control Infect, 2014;4:180-185.

25. SANTOS VB, et al. Infecção de sítio cirúrgico em mulheres submetidas à cesariana em uma maternidade pública. Revista Pesquisa em Saúde, 2017; 18: 35-40.

26. SCHNEID-KOFMAN N, et al. Risk factors for wound infection following cesarean deliveries. Revista International Journal of Gynecology \& Obstetrics, 2005; 90: 10-15.

27. SOUSA AFL, et al. Complicações pós-operatórias tardias em pacientes cirúrgicos: uma revisão integrativa. Revista Brasileira de Enfermagem, 2020; 73: 45-52.

28. ZIMMERMMANN JB, et al. Complicações puerperais associadas à via de parto. Revista Médica de Minas Gerais, 2009; 19: 109-16. 